\title{
A SIMPLE ANALYTIC PROOF OF AN INEQUALITY BY P. BUSER
}

\author{
M. LEDOUX
}

(Communicated by Peter $\mathrm{Li}$ )

\begin{abstract}
We present a simple analytic proof of the inequality of P. Buser showing the equivalence of the first eigenvalue of a compact Riemannian manifold without boundary and Cheeger's isoperimetric constant under a lower bound on the Ricci curvature. Our tools are the Li-Yau inequality and ideas of Varopoulos in his functional approach to isoperimetric inequalities and heat kernel estimates on groups and manifolds. The method is easily modified to yield a logarithmic isoperimetric inequality involving the hypercontractivity constant of the manifold.
\end{abstract}

\section{BUSER'S INEQUALITY}

Throughout this paper, $M$ will denote a compact Riemannian manifold without boundary of dimension $n$. We denote by $\mu$ the normalised Riemannian measure on $M$, by $\Delta$ the Laplace operator, and by $\nabla f$ the gradient of a smooth function $f$ on $M$ with Riemannian length $|\nabla f|$.

The first nontrivial eigenvalue $\lambda_{1}$ of the Laplacian is characterised via the min-max theorem by the Poincaré type inequality

$$
\lambda_{1} \int f^{2} d \mu \leq \int|\nabla f|^{2} d \mu
$$

holding for all smooth functions $f$ on $M$ with $\int f d \mu=0$. Alternatively, by the spectral theorem (or simply by differentiation),

$$
\left\|P_{t} f\right\|_{2} \leq e^{-\lambda_{1} t}\|f\|_{2}, \quad t \geq 0,
$$

for all $f$ with $\int f d \mu=0$, where $\|\cdot\|_{p}$ is the $L^{p}$-norm $(1 \leq p \leq \infty)$ with respect to $\mu$ and where $P_{t}=e^{t \Delta}, t \geq 0$, is the heat semigroup on $M$.

In 1970, Cheeger [C] introduced an isoperimetric constant to bound below the first eigenvalue $\lambda_{1}$. Set

$$
h=\inf \frac{a(\partial A)}{\mu(A)},
$$

where the infimum runs over all open subsets $A$ with $\mu(A) \leq \frac{1}{2}$ and smooth boundary $\partial A$, and where $a(\cdot)$ denotes the $(n-1)$-dimensional measure.

Received by the editors October 14, 1992.

1991 Mathematics Subject Classification. Primary 58G11, 58G25, 53C99, 49Q15. 
Cheeger's result is that

$$
\lambda_{1} \geq \frac{h^{2}}{4}
$$

One simple argument to derive (2) may be sketched as follows (see [Y, GHL]). First note that the definition of $h$ together with the coarea formula $[\mathrm{F}, \mathrm{C}]$ leads to

$$
h \int_{0}^{\infty} \min (\mu(g \geq s), 1-\mu(g \geq s)) d s \leq \int|\nabla g| d \mu
$$

for every positive smooth $g$ on $M$. Now, let $f$ be a smooth function on $M$ and denote by $m$ a median of $f$ for $\mu$, i.e., $\mu(f \geq m) \geq \frac{1}{2}$ and $\mu(f \leq m) \geq \frac{1}{2}$. Set $f^{+}=\max (f-m, 0), f^{-}=-\min (f-m, 0)$ so that $f-m=f^{+}-f^{-}$. By the definition of the median, for every $s>0$,

$$
\mu\left(\left(f^{+}\right)^{2} \geq s\right) \leq \frac{1}{2} \quad \text { and } \quad \mu\left(\left(f^{-}\right)^{2} \geq s\right) \leq \frac{1}{2} .
$$

Hence, (3) applied to $g=\left(f^{+}\right)^{2}$ and $g=\left(f^{-}\right)^{2}$ together with integration by parts yields

$$
\begin{aligned}
h \int|f-m|^{2} d \mu & =h \int\left(f^{+}\right)^{2} d \mu+h \int\left(f^{-}\right)^{2} d \mu \\
& =h \int_{0}^{\infty} \mu\left(\left(f^{+}\right)^{2} \geq s\right) d s+h \int_{0}^{\infty} \mu\left(\left(f^{-}\right)^{2} \geq s\right) d s \\
& \leq \int\left|\nabla\left(f^{+}\right)^{2}\right| d \mu+\int\left|\nabla\left(f^{-}\right)^{2}\right| d \mu .
\end{aligned}
$$

By the Cauchy-Schwarz inequality, the right-hand side of this inequality is less than

$$
2\left(\int|f-m|^{2} d \mu\right)^{1 / 2}\left(\int|\nabla f|^{2} d \mu\right)^{1 / 2} .
$$

Therefore, for every median $m$ of $f$,

$$
\frac{h^{2}}{4} \int|f-m|^{2} d \mu \leq \int|\nabla f|^{2} d \mu .
$$

Since the mean $\int f d \mu$ minimises $\int|f-c|^{2} d \mu, c \in \mathbb{R}$, inequality (2) immediately follows. If $M$ has a boundary, then Cheeger's inequality still holds if $\lambda_{1}$ is subject to the Neumann boundary condition.

Cheeger's inequality $\lambda_{1} \geq h^{2} / 4$ proved extremely useful in finding geometrical lower bounds on $\lambda_{1}$ via the isoperimetric constant $h$. It was therefore an important observation by Buser [B] that this inequality is sharp in the sense that $\lambda_{1}$ and $h$ are actually equivalent, with constants depending only on the dimension and the Ricci curvature of $M$. More precisely, Buser obtained the following result.

Theorem 1. Let $M$ be a compact Riemannian manifold without boundary whose Ricci curvature is bounded below by $-K, K \geq 0$. Then

$$
\lambda_{1} \leq C\left(\sqrt{K} h+h^{2}\right),
$$

where $C>0$ is a constant which depends only on the dimension of $M$.

Proof of Theorem 1. While the proof of Buser is geometric, the aim of this paper is to provide a simple analytic proof of this inequality using some semigroup 
techniques inspired from the work of Varopoulos [V1, V2] in his functional approach to isoperimetric inequalities and heat kernel estimates on groups and manifolds.

We present the basic idea of the proof first. Recall the heat semigroup $\left(P_{t}\right)_{t \geq 0}$. We start from the Li-Yau inequality [LY]: for every $f$ positive and smooth, and every $\alpha>1, t>0$, at each point of $M$,

$$
\frac{\left|\nabla P_{t} f\right|^{2}}{\left(P_{t} f\right)^{2}}-\alpha \frac{\Delta P_{t} f}{P_{t} f} \leq \frac{n \alpha^{2}}{2 t}\left(1+\frac{K t}{\alpha-1}\right) .
$$

We will use this inequality with simply, say, $\alpha=2$. Following [V2], this inequality implies that, for every $t_{0}>0,0<t \leq t_{0}$, and every $f$ positive and smooth,

$$
\left\|\left|\nabla P_{t} f\right|\right\|_{\infty} \leq \frac{C}{\sqrt{t}}\|f\|_{\infty},
$$

where $C=\left[3 n\left(1+K t_{0}\right)\right]^{1 / 2}$.

For the sake of completeness, we briefly recall below the proofs of (4) and (5), but first, we would like to describe how inequality (5) may be used to establish the theorem.

We assume in the following that $K>0$. When $K=0$, (4) actually holds with $\alpha=1$ and (5) for every $t>0$, so that the argument below is trivially modified to this case. Let us choose therefore $t_{0}=1 / K$ in (5) (hence $C=$ $\left.(6 n)^{1 / 2}\right)$. Integrating (5) yields, by duality, for every $f$ positive and smooth and every $0<t \leq 1 / K$,

$$
\left\|f-P_{t} f\right\|_{1} \leq 2 C \sqrt{t}\||\nabla f|\|_{1} .
$$

Indeed, for every $g$ smooth with $\|g\|_{\infty} \leq 1$,

$$
\begin{aligned}
\int g\left(f-P_{t} f\right) d \mu & =-\int_{0}^{t}\left(\int g \Delta P_{s} f d \mu\right) d s=\int_{0}^{t}\left(\int \nabla P_{s} g \cdot \nabla f d \mu\right) d s \\
& \leq\||\nabla f|\|_{1} \int_{0}^{t}\left\|\left|\nabla P_{s} g\right|\right\|_{\infty} d s \leq 2 C \sqrt{t}\||\nabla f|\|_{1},
\end{aligned}
$$

where we used (5) in the last step. Now, we simply apply inequality (6) to smooth functions approximating the characteristic function $\chi_{A}$ of an open set $A$ in $M$ with smooth boundary $\partial A$. It yields, for every $0<t \leq 1 / K$,

$$
\begin{aligned}
2 C \sqrt{t} a(\partial A) & \geq \int_{A}\left[1-P_{t}\left(\chi_{A}\right)\right] d \mu+\int_{A^{c}} P_{t}\left(\chi_{A}\right) d \mu \\
& =2\left(\mu(A)-\int_{A} P_{t}\left(\chi_{A}\right) d \mu\right)=2\left(\mu(A)-\left\|P_{t / 2}\left(\chi_{A}\right)\right\|_{2}^{2}\right) .
\end{aligned}
$$

Now, by (1),

$$
\left\|P_{t / 2}\left(\chi_{A}\right)\right\|_{2}^{2}=\mu(A)^{2}+\left\|P_{t / 2}\left(\chi_{A}-\mu(A)\right)\right\|_{2}^{2} \leq \mu(A)^{2}+e^{-\lambda_{1} t}\left\|\chi_{A}-\mu(A)\right\|_{2}^{2}
$$

so that, with the preceding,

$$
2 C \sqrt{t} a(\partial A) \geq 2 \mu(A)(1-\mu(A))\left(1-e^{-\lambda_{1} t}\right)
$$

for every $0<t \leq 1 / K$. Therefore,

$$
h \geq \frac{1}{2 C} \sup _{0<t \leq 1 / K}\left(\frac{1-e^{-\lambda_{1} t}}{\sqrt{t}}\right) .
$$


The proof is complete. Indeed, if $\lambda_{1} \geq K$, we can choose $t=1 / \lambda_{1}$ in the supremum of (9) to get

$$
h \geq \frac{1}{2 C}\left(1-\frac{1}{e}\right) \sqrt{\lambda_{1}},
$$

while if $\lambda_{1} \leq K$, we simply take $t=1 / K$ and then

$$
h \geq \frac{1}{2 C} \sqrt{K}\left(1-e^{-\lambda_{1} / K}\right) \geq \frac{1}{4 C} \cdot \frac{\lambda_{1}}{\sqrt{K}} .
$$

In any case,

$$
\lambda_{1} \leq 4 C \sqrt{K} h+16 C^{2} h^{2}
$$

which is the result.

As announced, and for the sake of completeness, we briefly recall, to conclude this proof, the steps (4) and (5) due, respectively, to Li and Yau [LY] and Varopoulos [V2].

Our exposition of the Li-Yau inequality follows [D]. The starting point is the Bochner formula (see [BGM, GHL]) which leads to the inequality

$$
\frac{1}{2} \Delta\left(|\nabla g|^{2}\right)-\nabla g \cdot \nabla(\Delta g) \geq \frac{1}{n}(\Delta g)^{2}-K|\nabla g|^{2}
$$

for all smooth functions $g$ on $M$. Now, let $f$ be positive and smooth and, on $M \times[0, T], T>0$, set $g=\log P_{t} f$. We observe that

$$
\Delta g+|\nabla g|^{2}=g_{t},
$$

where $g_{t}$ is differentiation with respect to time. By (10), it follows that

$$
\begin{aligned}
\frac{2}{n}(\Delta g)^{2}-2 K|\nabla g|^{2} & \leq \Delta\left(|\nabla g|^{2}\right)-2 \nabla g \cdot \nabla g_{t}+2 \nabla g \cdot \nabla\left(|\nabla g|^{2}\right) \\
& =\Delta\left(|\nabla g|^{2}\right)-\frac{d}{d t}|\nabla g|^{2}+2 \nabla g \cdot \nabla\left(|\nabla g|^{2}\right) .
\end{aligned}
$$

Multiply this inequality by $t$ and set $H=t|\nabla g|^{2}$ so that

$$
t\left[\frac{2}{n}(\Delta g)^{2}-2 K|\nabla g|^{2}\right] \leq \Delta H-H_{t}+\frac{H}{t}+2 \nabla g \cdot \nabla H .
$$

Now, if we let $I=t g_{t}$, it is elementary from (11) that

$$
\Delta I-I_{t}+\frac{I}{t}+2 \nabla g \cdot \nabla I=0 .
$$

Therefore, if, for any real number $\alpha$, we let $\left.G=H-\alpha I=\left.t(\mid \nabla g)\right|^{2}-\alpha g_{t}\right)$, we have obtained that, on $M \times[0, T]$,

$$
t\left[\frac{2}{n}(\Delta g)^{2}-2 K|\nabla g|^{2}\right] \leq \Delta G-G_{t}+\frac{G}{t}+2 \nabla g \cdot \nabla G .
$$

Let $(x, t)$ be a point in $M \times[0, T]$ at which $G$ takes its maximum value. Assume first that $G(x, t)>0$. Then $t>0$ and, at $(x, t), \nabla G=0, \Delta G \leq 0$, $G_{t} \geq 0$. Therefore, at $(x, t),(12)$ yields

$$
\frac{2}{n}(\Delta g)^{2} \leq \frac{G}{t^{2}}+2 K|\nabla g|^{2}
$$


Assume $\alpha>1$. Recall that $\Delta g=g_{t}-|\nabla g|^{2}$ and $G=t\left(|\nabla g|^{2}-\alpha g_{t}\right)$. Thus

$$
\frac{2}{n}\left[\left(1-\frac{1}{\alpha}\right)|\nabla g|^{2}+\frac{G}{\alpha t}\right]^{2} \leq \frac{G}{t^{2}}+2 K|\nabla g|^{2} \text {. }
$$

Multiply both sides of this inequality by $\alpha^{2} t^{2}$ and set $J=|\nabla g|^{2} / G$. Simplifying by $G$ then yields

$$
\frac{2}{n}(1+(\alpha-1) t J)^{2} G \leq \alpha^{2}\left(1+2 K t^{2} J\right)
$$

Hence, at $(x, t)$,

$$
G \leq \frac{n \alpha^{2}}{2} \cdot \frac{1+2 K t^{2} J}{[1+(\alpha-1) t J]^{2}} \leq \frac{n \alpha^{2}}{2}\left(1+\frac{K t}{\alpha-1}\right) .
$$

This inequality also holds when $G(x, t) \leq 0$, and, recalling that $g=\log P_{t} f$, is exactly (4).

To deduce (5) from (4), let thus $\alpha=2$ and note that (4) implies that, for every $0<t \leq t_{0}$ and every $f$ positive and smooth,

$$
\left(\Delta P_{t} f\right)^{-} \leq n\left(1+K t_{0}\right) \frac{1}{t} P_{t} f
$$

where $(\cdot)^{-}$is the negative part. Since $\int \Delta P_{t} f d \mu=0$ and $\int P_{t} f d \mu=\int f d \mu$,

$$
\left\|\Delta P_{t} f\right\|_{1} \leq \frac{2 C^{\prime}}{t}\|f\|_{1}
$$

with $C^{\prime}=n\left(1+K t_{0}\right)$. By duality, for every $f$ and $t>0$,

$$
\left\|\Delta P_{t} f\right\|_{\infty} \leq \frac{2 C^{\prime}}{t}\|f\|_{\infty}
$$

Coming back to the Li-Yau inequality (4),

$$
\left\|\left|\nabla P_{t} f\right|\right\|_{\infty}^{2} \leq \frac{3 C^{\prime}}{t}\|f\|_{\infty}^{2}
$$

which is $(5)$.

The preceding proof of Theorem 1 extends to complete noncompact manifolds. Define the bottom of the spectrum $\lambda_{1}$ as the infimum of $\int|\nabla f|^{2} d \mu \mid$ $\int f^{2} d \mu$, where $f$ runs over sufficiently smooth functions with compact support (assume the volume of $M$ is infinite). Let $h$ be as before but with the additional condition that $A \cup \partial A$ be compact. Since (4) and (5) hold similarly in the noncompact case, one gets in the same way from (6), (7), and (8) that for every open subset $A$ such that $A \cup \partial A$ is compact and all $0<t \leq 1 / K$,

$$
2 C \sqrt{t} a(\partial A) \geq 2\left(\mu(A)-\left\|P_{t / 2}\left(\chi_{A}\right)\right\|_{2}^{2}\right) \geq 2 \mu(A)\left(1-e^{-\lambda_{1} t}\right) .
$$

The proof is then completed in the same way. Since, as mentioned in [B], $h \leq$ $\sqrt{(n-1) K}$ in the noncompact case, the final result here is that $\lambda_{1} \leq C \sqrt{K} h$, where $C$ only depends on the dimension.

\section{ON A LOGARITHMIC ISOPERIMETRIC INEQUALITY}

It is a simple matter to modify the preceding approach by using other geometric invariants of $M$ instead of the spectral gap $\lambda_{1}$. One may use, as in [V1, V2, 
Co], Sobolev constants via heat kernel decays. One may also use the so-called hypercontractivity constant $\rho_{0}$ of the Laplace operator on $M$ defined as the least $\rho>0$ such that whenever $1<p<q<\infty$ and $e^{\rho t} \geq[(q-1) /(p-1)]^{1 / 2}$,

$$
\left\|P_{t} f\right\|_{q} \leq\|f\|_{p}
$$

for every $f$ on $M$ (in $L^{p}(\mu)$ ). It is known [R1] that on every compact Riemannian manifold $\lambda_{1} \geq \rho_{0}>0$, and that [Gr] (13) may be expressed equivalently as a logarithmic Sobolev inequality

$$
\rho_{0}\left[\int f^{2} \log |f| d \mu-\int f^{2} d \mu \log \left(\int f^{2} d \mu\right)^{1 / 2}\right] \leq \int|\nabla f|^{2} d \mu
$$

for all smooth functions $f$ on $M$.

Now, if we follow the proof of Theorem 1 and replace, in (7), (8), the spectral estimate by the hypercontractivity inequality (13), we simply get that, for all open subsets $A$ of $M$ with smooth boundary $\partial A$ and for all $0<t \leq 1 / K$,

$$
2 C \sqrt{t} a(\partial A) \geq 2\left(\mu(A)-\left\|P_{t / 2}\left(\chi_{A}\right)\right\|_{2}^{2}\right) \geq 2\left(\mu(A)-\mu(A)^{2 / p(t)}\right),
$$

where $p(t)=1+e^{-\rho_{0} t}\left(\right.$ and $\left.C=(12 n)^{1 / 2}\right)$. Set $t_{0}=\min \left(1 / K, 1 / \rho_{0}\right)$. Since $1-e^{-x} \geq x / 2$ for $0 \leq x \leq 1$, it follows that for every $0<t \leq t_{0}$,

$$
C \sqrt{t} a(\partial A) \geq \mu(A)\left(1-\mu(A)^{\rho_{0} t / 4}\right)=\mu(A)\left[1-\exp \left(-\frac{\rho_{0} t}{4} \log \frac{1}{\mu(A)}\right)\right]
$$

Assume $\mu(A)>0$. Choose then $0<t \leq t_{0}$ such that

$$
t=4 t_{0}\left(\log \frac{1}{\mu(A)}\right)^{-1}
$$

provided $\mu(A)$ is small enough so that $\mu(A) \leq e^{-4}$. For this value of $t,(15)$ reads as

$$
\begin{aligned}
a(\partial A) & \geq \frac{1}{2 C \sqrt{t_{0}}}\left(1-e^{-\rho_{0} t_{0}}\right) \mu(A)\left(\log \frac{1}{\mu(A)}\right)^{1 / 2} \\
& \geq \frac{1}{4 C} \rho_{0} \sqrt{t_{0}} \mu(A)\left(\log \frac{1}{\mu(A)}\right)^{1 / 2}
\end{aligned}
$$

since $\rho_{0} t_{0} \leq 1$.

The preceding inequality holds for $\mu(A) \leq e^{-4}$. In general however, when $0 \leq \mu(A) \leq \frac{1}{2}$, we can always apply (15) with $t=t_{0}$ to get

$$
a(\partial A) \geq \frac{1}{C \sqrt{t_{0}}} \mu(A)\left[1-\exp \left(-\frac{\rho_{0} t_{0}}{4} \log 2\right)\right] \geq \frac{1}{16 C} \rho_{0} \sqrt{t_{0}} \mu(A),
$$

so that, combined with the preceding, for every $A$ with $0 \leq \mu(A) \leq \frac{1}{2}$,

$$
a(\partial A) \geq \frac{1}{32 C} \rho_{0} \sqrt{t_{0}} \mu(A)\left(\log \frac{1}{\mu(A)}\right)^{1 / 2} .
$$

Hence, we have established the following theorem. 
Theorem 2. Let $M$ be a compact Riemannian manifold without boundary whose Ricci curvature is bounded below by $-K, K \geq 0$. Then, if $\rho_{0}$ denotes the hypercontractivity constant of $M$, for every open subset $A$ of $M$ with $0 \leq \mu(A) \leq \frac{1}{2}$ and smooth boundary $\partial A$,

$$
a(\partial A) \geq \frac{1}{C} \min \left(\frac{\rho_{0}}{\sqrt{K}}, \sqrt{\rho_{0}}\right) \mu(A)\left(\log \frac{1}{\mu(A)}\right)^{1 / 2},
$$

where $C$ only depends on the dimension of $M$.

While only of logarithmic type with respect to the results of [G, B-B-G], this isoperimetric inequality, on the other hand, involves $\rho_{0}$ rather than the diameter of the manifold. The isoperimetric function in Theorem 2 is precisely (a form of) the isoperimetric function in Gaussian space (cf. [L]). On the basis of Theorem 2, one may thus conjecture some "infinite dimensional" extension of the Lévy-Gromov isoperimetric inequality of $[G]$ which would compare, independently of the dimension, the isoperimetric property of a diffusion generator (with positive curvature) to the Gaussian isoperimetric inequality. In the context of Theorem 2, this would amount to showing that the constant $C$ may actually be chosen independent of the dimension of the manifold. Going back to the proof, we would need the constant in (6) or (5) to be independent of $n$.

Theorem 2 may actually be stated in an equivalent formulation close to Buser's inequality. Define the logarithmic isoperimetric constant $k$ of $M$ as the infimum of

$$
\frac{a(\partial A)}{\mu(A)\left(\log \frac{1}{\mu(A)}\right)^{1 / 2}}
$$

over all open subsets $A$ with not more than half of the volume and smooth boundary $\partial A$. Note that clearly $h \geq k / 2$. One may compare $k$ to $\rho_{0}$ as in Cheeger's inequality. Namely, let $g$ be positive and smooth on $M$. Then, by the coarea formula,

$$
k \int_{s_{0}}^{\infty} \mu(g \geq s)\left(\log \frac{1}{\mu(g \geq s)}\right)^{1 / 2} d s \leq \int|\nabla g| d \mu,
$$

where $s_{0}$ is such that $\mu\left(g \geq s_{0}\right) \leq \frac{1}{2}$. Let now $f$ be smooth with $\int f^{2} d \mu=1$ and apply the preceding inequality to $g=f^{2}\left(\log \left(3+f^{2}\right)\right)^{1 / 2}$. After some elementary, but tedious, computations, we get

$$
\int f^{2} \log \left(3+f^{2}\right) d \mu \leq \frac{\alpha}{k^{2}} \int|\nabla f|^{2} d \mu+\alpha
$$

for some numerical constant $\alpha>0$. For any function $f$ on $M$, set

$$
E(f)=\int f^{2} \log |f| d \mu-\int f^{2} d \mu \log \left(\int f^{2} d \mu\right)^{1 / 2} .
$$

So far, we have obtained by homogeneity that, for some numerical constant $\alpha$ (not necessarily the same at each occurrence) and every smooth function $f$ on $M$,

$$
E(f) \leq \alpha\left(\frac{1}{k^{2}} \int|\nabla f|^{2} d \mu+\int f^{2} d \mu\right) .
$$


This is not yet (14), and to get rid of the extra factor, we may use the spectral gap $\lambda_{1}$. Namely, by [DS, p. 246] or [R2], we know that for every $f$,

$$
E(f) \leq E\left(f-\int f d \mu\right)+\int\left|f-\int f d \mu\right|^{2} d \mu .
$$

Hence, (16) applied to $f-\int f d \mu$ combined with this inequality yields

$$
\begin{aligned}
E(f) & \leq \frac{\alpha}{k^{2}} \int|\nabla f|^{2} d \mu+(\alpha+1) \int\left|f-\int f d \mu\right|^{2} d \mu \\
& \leq\left(\frac{\alpha}{k^{2}}+\frac{\alpha+1}{\lambda_{1}}\right) \int|\nabla f|^{2} d \mu
\end{aligned}
$$

for every smooth $f$ on $M$. Since $h \geq k / 2$, and hence $\lambda_{1} \geq k^{2} / 16$, it follows that $\rho_{0} \geq k^{2} / \alpha$ for some numerical constant $\alpha>0$. This relation clarifies and improves parts of [R2].

On the other hand, the content of Theorem 2 is that

$$
\rho_{0} \leq C\left(k \sqrt{K}+k^{2}\right),
$$

where $C$ only depends on the dimension.

\section{ACKNOWLEDGMENT}

I am grateful to Th. Coulhon for helpful comments.

\section{REFERENCES}

[BBG] P. Bérard, G. Besson, and S. Gallot, Sur une inégalité isopérimétrique qui généralise celle de Paul Lévy-Gromov, Invent. Math. 80 (1985), 295-308.

[BGM] M. Berger, P. Gauduchon, and $\mathrm{E}$. Mazet, Le spectre d'une variété riemannienne, Lecture Notes in Math., vol. 194, Springer-Verlag, Berlin and New York, 1971.

[B] P. Buser, A note on the isoperimetric constant, Ann. Sci. École Norm. Sup. 15 (1982), 213-230.

[C] J. Cheeger, $A$ lower bound for the smallest eigenvalue of the Laplacian, Problems in Analysis, Symposium in honor of S. Bochner, Princeton Univ. Press, Princeton, NJ, 1970, pp. 195-199.

[Co] Th. Coulhon, Sobolev inequalities on graphs and on manifolds, Harmonic Analysis and Discrete Potential Theory (M. Picardello, ed.), Plenum Press, New York, 1992, pp. 207-214.

[D] E. B. Davies, Heat kernels and spectral theory, Cambridge Univ. Press, London and New York, 1989.

[DS] J.-D. Deuschel and D. Stroock, Large deviations, Academic Press, New York, 1989.

[F] H. Federer, Geometric measure theory, Springer-Verlag, Berlin and New York, 1969.

[GHL] S. Gallot, D. Hulin, and J. Lafontaine, Riemannian geometry, 2nd ed., Springer-Verlag, Berlin and New York, 1990.

[G] M. Gromov, Paul Lévy's isoperimetric inequality, Inst. Hautes Etudes Sci., preprint, 1980.

[Gr] L. Gross, Logarithmic Sobolev inequalities, Amer. J. Math. 97 (1975), 1061-1083.

[L] M. Ledoux, Semigroup proofs of the isoperimetric inequality in Euclidean and Gauss space, Bull. Sci. Math. (2) (1992) (to appear).

[LY] P. Li and S.-T. Yau, On the parabolic kernel of the Schrödinger operator, Acta Math. 156 (1986), 153-201.

[R1] O. S. Rothaus, Diffusion on compact Riemannian manifolds and logarithmic Sobolev inequalities, J. Funct. Anal. 42 (1981), 102-109.

[R2] - Analytic inequalities, isoperimetric inequalities and logarithmic Sobolev inequalities, J. Funct. Anal. 64 (1985), 296-313. 
[V1] N. Varopoulos, Hardy-Littlewood theory for semigroups, J. Funct. Anal. 63 (1985), 240-260.

[V2] Small time Gaussian estimates of heat diffusion kernels, I, The semigroup technique, Bull. Sci. Math. (2) 113 (1989), 253-277.

[Y] S.-T. Yau, Isoperimetric constants and the first eigenvalue of a compact Riemannian manifold, Ann. Sci. École Norm. Sup. 8 (1975), 487-507.

Département de Mathématiques, Université Paul-Sabatier, 31062 Toulouse, France 